\title{
Investigation of General Power Sum-Connectivity Index for Some Classes of Extremal Graphs
}

\author{
Rui Cheng, ${ }^{1}$ Gohar Ali $\mathbb{D}^{2},{ }^{2}$ Gul Rahmat, ${ }^{2}$ Muhammad Yasin Khan, \\ Andrea Semanicova-Fenovcikova $\mathbb{D D}^{3}$ and Jia-Bao Liu $\mathbb{D}^{4}$ \\ ${ }^{1}$ School of Resources and Environment, Anhui Agricultural University, Hefei 230036, China \\ ${ }^{2}$ Department of Mathematics, Islamia College, Peshawar, Khyber Pakhtunkhwa, Pakistan \\ ${ }^{3}$ Department of Applied Mathematics and Informatics, Faculty of Mechanical Engineering, Technical University in Kosice, \\ Letna 9, Kosice, Slovakia \\ ${ }^{4}$ School of Mathematics and Physics, Anhui Jianzhu University, Hefei 230601, China \\ Correspondence should be addressed to Gohar Ali; gohar.ali@icp.edu.pk
}

Received 23 June 2021; Accepted 6 August 2021; Published 23 August 2021

Academic Editor: Muhammad Javaid

Copyright ( $) 2021$ Rui Cheng et al. This is an open access article distributed under the Creative Commons Attribution License, which permits unrestricted use, distribution, and reproduction in any medium, provided the original work is properly cited.

\begin{abstract}
In this work, we introduce a new topological index called a general power sum-connectivity index and we discuss this graph invariant for some classes of extremal graphs. This index is defined by $Y_{\alpha}(G)=\sum_{u v \in E(G)}\left(d(u)^{d(u)}+d(v)^{d(v)}\right)^{\alpha}$, where $d(u)$ and $d(v)$ represent the degree of vertices $u$ and $v$, respectively, and $\alpha \geq 1$. A connected graph $G$ is called a $k$-generalized quasi-tree if there exists a subset $V_{k} \subset V(G)$ of cardinality $k$ such that the graph $G-V_{k}$ is a tree but for any subset $V_{k-1} \subset V(G)$ of cardinality $k-1$, the graph $G-V_{k-1}$ is not a tree. In this work, we find a sharp lower and some sharp upper bounds for this new sumconnectivity index.
\end{abstract}

\section{Introduction}

In this article, all the graphs are considered as simple, connected, finite, and undirected. Let us denote a graph by $G=(V(G), E(G))$, where $V(G)$ and $E(G)$ represent the sets of vertices and edges, respectively. Degree of vertex $u$ is the number of adjacent vertices to $u$ and is denoted by $d(u)$, and the set of vertices adjacent to the vertex $u$ is denoted by $N(u)$. The length of a shortest path between two vertices, say $u$ and $v$, is termed as the distance between these vertices and is denoted by $d(u, v)$. The maximum distance from vertex $u$ to any other vertex is known as the eccentricity of the vertex $u$ and is denoted by $\varepsilon(u)$ and defined as $\mathcal{E}(u)=\max _{v \in V(G)} d(u, v)$. The diameter of a graph $G$ is $\max _{u \in V(G)}=\max _{u, v \in V(G)}$, and its notation is $\operatorname{diam}(G)$; further, see $[1-3]$.

Let $K_{n}$ be a complete graph of order $n$. The complete bipartite graph $K_{1, n-1}$, which is also denoted as $S_{n}$, represents a star of order $n$, while $P_{n}$ is the path of order $n$ and size $n-1$. A double star of order $a+b$ is denoted by $S_{a, b}$. The graph $S_{a, b}$ is a tree which consists of two adjacent vertices say $u$ and $v$, such that $u$ is adjacent to $b-1$ pendent vertices and $v$ is adjacent to $a-1$ pendent vertices. Alternatively, the double star $S_{a, b}$ can be obtained by joining the centers of two stars $S_{a+1}$ and $S_{b+1}$. If for a tree $T$ the diameter of $T$ is 2 , then $T$ is a star graph, and if the diameter of $T$ is 3 , then $T$ is a double star. For two graphs $G$ and $H$ whose vertex sets are disjoint, $G+H$ denotes its join graph having the vertex set $V(G+$ $H)=V(G) \cup V(H)$ and $E(G+H)=E(G) \cup E(H) \cup$ $\{u v: u \in V(G), v \in V(H)\}$ is its edge set.

If there exists a vertex $v$ in a graph $G$ such that the graph $G-V$ is a tree, then the graph $G$ is called a quasi-tree and the vertex $v$ is called a quasi-vertex. Obviously, every tree is a quasi-tree, as by deletion of any vertex in a tree, the resulting graph is again a tree. Furthermore, a $k$-generalized quasi-tree is a graph in which there exists a subset $V_{k} \subset V(G)$ with cardinality $k$ such that the graph $G-V_{k}$ is a tree but for any subset $V_{k-1} \subset V(G)$ with cardinality $k-1$, the graph $G-$ $V_{k-1}$ is not a tree. The vertices in the set $V_{k}$ are called $k$-quasi-vertices or quasi-vertices. We need at least $k+2$ 
vertices for sketching $k$-generalized quasi-tree. Any tree is a quasi-tree which is trivial. Let the class of $k$-generalized quasi-trees of order $n$ be denoted by $T_{k}(n)$.

In this modern world, the network structure plays the basic role in the field of chemistry, technology, and communication. Every network is distinguished by means of numerical quantity under some parameter. Such roles are called topological indices. A numerical quantity that is invariant under graph automorphisms is termed as the topological index. There are many topological indices such as degree-based, distance-based, and counting-related topological indices. In all the said indices, degree-based topological indices are one of the basic indices and play the key role in chemistry and chemical graph theory. The mostly studied topological indices are the atom-bond $(\mathrm{ABC})$ connectivity index, the harmonic $(\mathrm{H})$ index, the Zagreb index, and various others; further we refer to [4-10].

On the basis of the previously defined indices, we introduce a new sum-connectivity index called a general power sum-connectivity index defined such that

$$
Y_{\alpha}(G)=\sum_{u v \in E(G)}\left(d(u)^{d(u)}+d(v)^{d(v)}\right)^{\alpha}, \quad \alpha \geq 1 .
$$

\section{Main Work}

This section is devoted to the main results which we proved for the new introduced sum-connectivity index for some classes of extremal graphs. The following are the main results in this regard.

Lemma 1. For any two vertices $u$ and $v \in V(G)$, where $u v \notin E(G)$, then

$$
Y_{\alpha}(G)<Y_{\alpha}(G+u v) .
$$

Theorem 1. The general power sum-connectivity index $Y_{\alpha}(G)$ is minimum for a graph $G$ if $G=P_{n}$, for $n \geq 4$ and $\alpha \geq 1$.

Proof. Let $G$ be the graph of order $n$. To prove that $Y_{\alpha}(G)$ is minimum for $G=P_{n}$, where $n \geq 4$, we consider two cases.

Case 1. When $n=4$, in this case, the graph is isomorphic either to a path $P_{4}$ or to a star $S_{4}$, see Figure 1.

In this case, we have

$$
Y_{\alpha}\left(P_{4}\right)=2 \cdot 5^{\alpha}+8^{\alpha}
$$

and similarly,

$$
Y_{\alpha}\left(S_{4}\right)=3 \cdot(28)^{\alpha} .
$$

It is obvious that $Y_{\alpha}\left(P_{4}\right)<Y_{\alpha}\left(S_{4}\right)$. Equality holds if $\alpha=0$. This can be easily analyzed through the following plot illustrated on Figure 2.

Case 2. When $n \geq 5$, in this case, suppose on contrary that $Y_{a}\left(P_{n}\right)$ is not minimum. Then, there exists a graph other

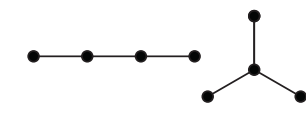

Figure 1: A path $P_{4}$ and a star $S_{4}$.

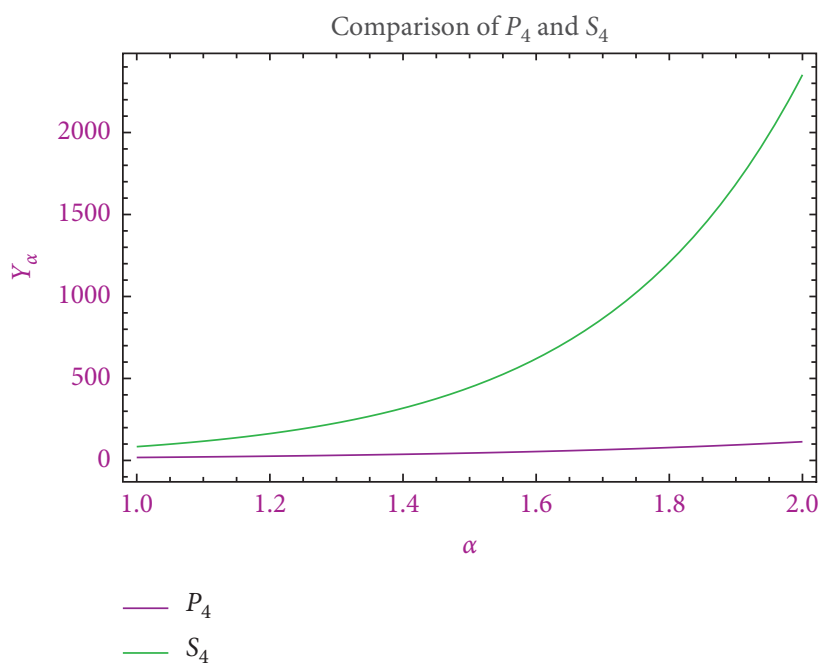

Figure 2: Plot for $Y_{\alpha}\left(P_{4}\right)$ and $Y_{\alpha}\left(S_{4}\right)$.

than $P_{n}$ in which at least one vertex, say $y$, has degree greater than 2 , i.e., such that $d_{G}(y) \geq 3$. We have to discuss the following subcases .

Subcase B1: when $y$ is adjacent to two leaves. A graph for this case is shown in Figure 3.

Here, we have

$$
Y_{\alpha}(G)=5^{\alpha}+(n-5) \cdot 8^{\alpha}+(31)^{\alpha}+2 \cdot(28)^{\alpha},
$$

and for $P_{n}$, we have

$$
Y_{\alpha}\left(P_{n}\right)=2 \cdot 5^{\alpha}+(n-3) \cdot 8^{\alpha} .
$$

From (5) and (6), this is obvious that $Y_{\alpha}(G)>Y_{\alpha}\left(P_{n}\right)$ which can be determined through the following plots illustrated on Figure 4 for various values of $n$.

This is a contradiction to the minimality of $Y_{\alpha}(G)$. Hence, in this case, $Y_{\alpha}\left(P_{n}\right)$ is minimal.

Subcase B2: when $y$ is adjacent to only one leaf. In this case, the graph is illustrated in Figure 5.

Here, $Y_{\alpha}(G)$ is given below:

$$
\begin{aligned}
Y_{\alpha}(G)= & \left(1+2^{2}\right)^{\alpha}+\left(2^{2}+3^{3}\right)^{\alpha}+\left(2^{2}+3^{3}\right)^{\alpha}+\left(1+3^{3}\right)^{\alpha} \\
& +\left(2^{2}+2^{2}\right)^{\alpha}+\left(2^{2}+2^{2}\right)^{\alpha}+\cdots+\left(1+2^{2}\right)^{\alpha} \\
= & 2 \cdot 5^{\alpha}+2 \cdot(31)^{\alpha}+(n-6) \cdot 8^{\alpha}+28^{\alpha} .
\end{aligned}
$$

Obviously, from (6) and (7), we have $Y_{\alpha}(G)>Y_{\alpha}\left(P_{n}\right)$ which can be observed through the following plots, see Figure 6, for various values of $n$, which is a contradiction to the minimality of $Y_{\alpha}(G)$. 


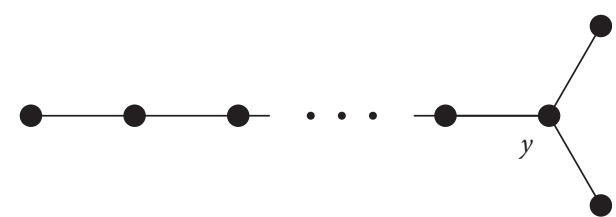

Figure 3: A graph $G$ with a vertex $y$ adjacent to two leaves.

Lemma 2. Let $G \in T_{k}(n)$, where $T_{k}(n)$ is the class of $k$-generalized quasi-trees. If $Y_{\alpha}(G)$ is maximum and $w$ is a quasi-vertex of $G$, then $d_{G}(w)=n-1$.

Proof. Consider that $G$ is a $k$-generalized quasi-tree from $T_{k}(n)$, where $Y_{\alpha}(G)$ is maximum for $G$ and let $w$ be a quasivertex of $G$. We show that $d_{G}(w)=n-1$. On contrary, suppose that $Y_{\alpha}(G)$ is maximum for $G$ and $d_{G}(w)<n-1$. Then, there is a vertex $f$ which is not adjacent to $w$. Then, by means of Lemma 1 , we have

$$
Y_{\alpha}(G)<Y_{\alpha}(G+w f),
$$

which is a contradiction to the maximality of $Y_{\alpha}(G)$. Hence, we are forced to accept that $d_{G}(w)=n-1$.

Roughly speaking that, for $\alpha \geq 1$, we define the following function:

$$
F\left(z_{1}, z_{2}, z_{3}, \ldots, z_{j}\right)=z_{1}^{\alpha \cdot z_{1}}+z_{2}^{\alpha \cdot z_{2}}+z_{3}^{\alpha \cdot z_{3}}+\cdots+z_{j}^{\alpha \cdot z_{j}},
$$

which is defined for $\left(z_{1}, z_{2}, z_{3}, \ldots, z_{j}\right) \in H_{j, q}$, where $H_{j, q}$ is a set containing vectors $\left(z_{1}, z_{2}, z_{3}, \ldots, z_{j}\right)$ having coordinates from positive integers and satisfying the following:

$$
\begin{aligned}
z_{1} \geq z_{2} \geq z_{3} \geq \cdots \geq z_{j} \geq 1, \\
q=\sum_{i=1}^{j} z_{i} .
\end{aligned}
$$

Further, for $1 \leq i<k \leq j$ and $z_{k} \geq 2$, we replace the components of $\left(z_{1}, z_{2}, \ldots, z_{j}\right)$ by $\left(z_{1}, z_{2}, \ldots, z_{i}+\right.$ $\left.1, \ldots, z_{k}-1, \ldots, z_{j}\right)$, rearranging the replaced components in decreasing order as

$$
\left(z_{1}^{*}, z_{2}^{*}, z_{3}^{*}, \ldots, z_{j}^{*}\right) \text {. }
$$

Let $\left(z_{1}^{*}, z_{2}^{*}, z_{3}^{*}, \ldots, z_{j}^{*}\right)=z^{*}$; then, obviously $z^{*} \in H_{j, q}$. Let this transformation be denoted by $A_{1}$. Next, we have

$$
F\left(z^{*}\right)-F\left(z_{1}\right)=\left(z_{i}+1\right)^{\alpha \cdot\left(z_{i}+1\right)}+\left(z_{k}-1\right)^{\alpha \cdot\left(z_{k}-1\right)}-z_{i}^{\alpha \cdot z_{i}}-z_{k}^{\alpha \cdot z_{k}} .
$$

We define another function:

$$
\psi(z)=z^{\alpha \cdot z}-(1+z)^{\alpha \cdot(1+z)} .
$$

This function is a strictly decreasing function for $z>0$ and $\alpha \geq 1$. Thus, if $i<k$, then $z_{i} \geq z_{k}$ which implies that
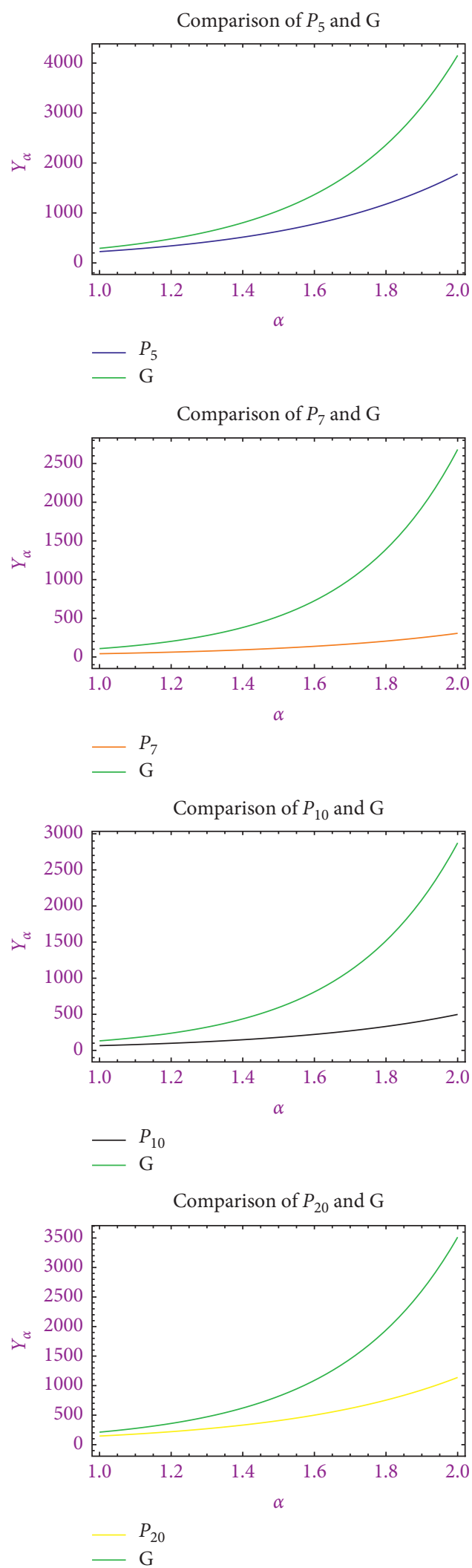

Figure 4: Plot for $Y_{\alpha}(G)$ and $Y_{\alpha}\left(P_{n}\right)$ for various values of $n$. 


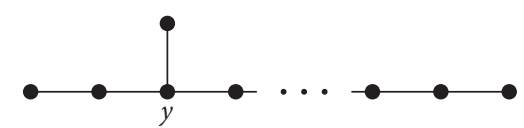

Figure 5: A graph $G$ with a vertex $y$ adjacent to one leaf.

$z_{k}-1<z_{i}$, and thus, $\psi\left(z_{k}-1\right)>\psi\left(z_{i}\right)$. This is due the decreasing function. We can also obtain that

$$
\begin{aligned}
& \left(z_{k}-1\right)^{\alpha \cdot\left(z_{k}-1\right)}-z_{k}^{\alpha \cdot z_{k}}>z_{i}^{\alpha \cdot z_{i}}-\left(1+z_{i}\right)^{\alpha \cdot\left(1+z_{i}\right),} \\
& \left(z_{k}-1\right)^{\alpha \cdot\left(z_{k}-1\right)}+\left(1+z_{i}\right)^{\alpha \cdot\left(1+z_{i}\right)}>z_{i}^{\alpha \cdot z_{i}}+z_{k}^{\alpha \cdot z_{k}} .
\end{aligned}
$$

We obtained that $F\left(z_{*}\right)-F\left(z_{1}, z_{2}, \ldots, z_{j}\right)>0$. We can easily deduce the following result.

Lemma 3. If there is an $2 \leq k \leq j$ having $x_{k} \geq 2$, then $F\left(z_{1}, z_{2}, \ldots, z_{j}\right)$ is strictly decreasing on $H_{j, q}$ having $\alpha \geq 1$.

In other words, we observe that the function is strictly increased if we push one unity to the left in the degree sequence and arrange this new sequence in decreasing order using the A1 transformation.

Theorem 2. Let $T$ be a tree in $T_{n}$, where $T_{n}$ is the class of trees of order $n$. Then, $Y_{\alpha}(T)$ is maximum if $T$ is the star graph $S_{n}$.

Proof. Let $T \in T_{n}$, such that $Y_{\alpha}(T)$ is maximum. We need to prove that $T=S_{n}$. For this proof, suppose on contrary that $Y_{\alpha}(T)$ is maximum and $T$ is neither a star $S_{n}$ nor a double star. Then, there is a vertex in $T$, say $t$, which is adjacent to two vertices $x$ and $y$, such that $d_{T}(x)=a, d_{T}(y)=b$, and $d_{T}(t)=c$, where $a$ and $b \geq 2$. Without loss of generality, let $a \geq b$, see Figure 7 .

Furthermore, for the neighbors of $x$ and $y$, we have $N(x)-t=\left\{x_{1}, x_{2}, \ldots, x_{a-1}\right\} \quad$ and $\quad N(y)-t=\left\{y_{1}\right.$, $\left.y_{2}, \ldots, y_{b-1}\right\}$. We can construct another tree $T^{*}$ from $T$ in such a way that we delete the edges $y y_{1}, y y_{2}, \ldots, y y_{b-1}$ and we insert new edges $x y_{1}, x y_{2}, \ldots, x y_{b-1}$ as in [11]. Now, we have the following:

$$
\begin{aligned}
Y_{\alpha}\left(T^{*}\right)= & \sum_{i=1}^{a-1}\left((a+b-1)^{a+b-1}+d_{T^{*}}\left(x_{i}\right)^{d_{T^{*}}\left(x_{i}\right)}\right)^{\alpha} \\
& +\sum_{i=1}^{b-1}\left((a+b-1)^{a+b-1}+d_{T^{*}}\left(y_{i}\right)^{d_{T^{*}}\left(y_{i}\right)}\right)^{\alpha} \\
& +\left((a+b-1)^{a+b-1}+c^{c}\right)^{\alpha}+\left(c^{c}+1\right)^{\alpha} .
\end{aligned}
$$

Similarly, for $T$, we have

$$
\begin{aligned}
Y_{\alpha}(T)= & \sum_{i=1}^{a-1}\left(a+d_{T}\left(x_{i}\right)^{d_{T}\left(x_{i}\right)}\right)^{\alpha}+\sum_{i=1}^{b-1}\left(b+d_{T}\left(y_{i}\right)^{d_{T}\left(y_{i}\right)}\right)^{\alpha} \\
& +\left(a^{a}+c^{c}\right)^{\alpha}+\left(c^{c}+b^{b}\right)^{\alpha} .
\end{aligned}
$$
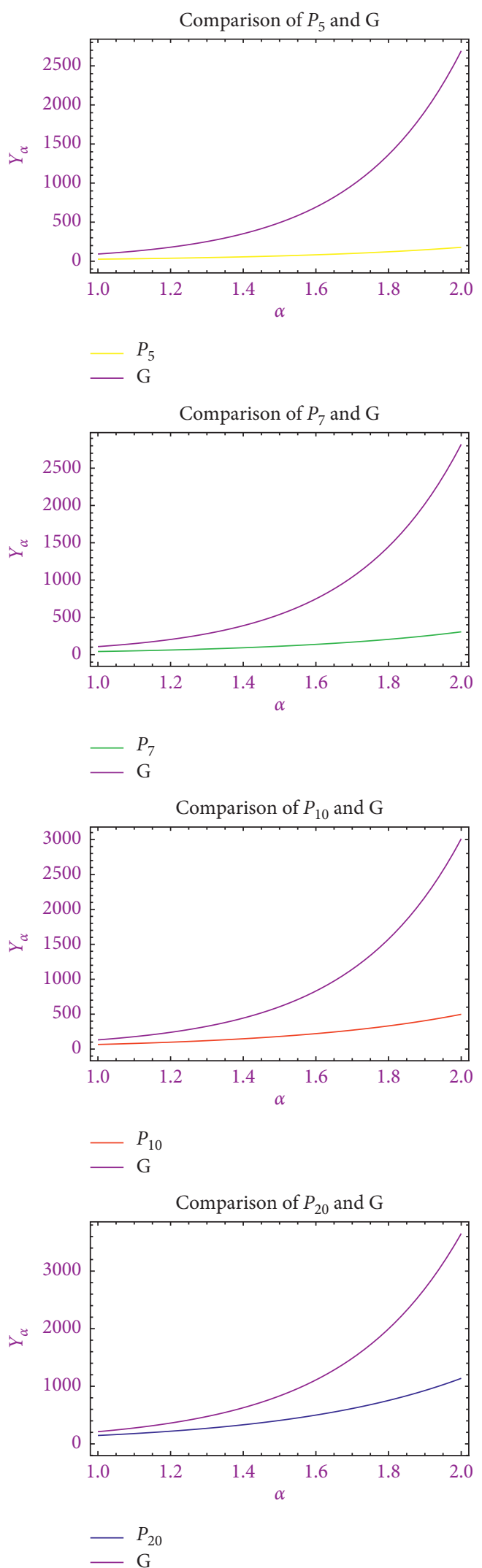

FiguRe 6: Plot for $Y_{\alpha}(G)$ and $Y_{\alpha}\left(P_{n}\right)$ for various values of $n$. 


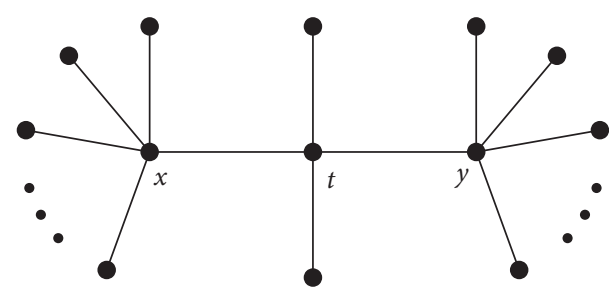

Figure 7: A tree $T$.

Next, we get the following:

$$
\begin{aligned}
Y_{\alpha}\left(T^{*}\right)-Y_{\alpha}(T)> & \left((a+b-1)^{a+b-1}+c^{c}\right)^{\alpha}+\left(c^{c}+1\right)^{\alpha} \\
& -\left(a^{a}+c^{c}\right)^{\alpha}-\left(c^{c}+b^{b}\right)^{\alpha}>0 .
\end{aligned}
$$

As a consequence of Lemma 3, for $j=2$ and $z_{1}=a^{a}+c^{c}$, $z_{2}=c^{c}+b^{b}, q=a^{a}+b^{b}+2 \cdot c^{c}$, and applying the transformation $A 1$ several times, we get a contradiction to the maximality of $Y_{\alpha}(T)$. Now, we show that, for $a$ and $b \geq 2$ and $a+b=n$, we have the following:

$$
Y_{\alpha}\left(S_{n}\right)>Y_{\alpha}\left(S_{a, b}\right)
$$

For $S_{n}$ and $S_{a, b}$, we obtain

$$
\begin{aligned}
Y_{\alpha}\left(S_{n}\right)= & (n-1)\left((n-1)^{n-1}+1\right)^{\alpha}, \\
Y_{\alpha}\left(S_{a, b}\right)= & (a-1)\left((a-1)^{a-1}+1\right)^{\alpha} \\
& +(b-1)\left((b-1)^{b-1}+1\right)^{\alpha}+\left(a^{a}+b^{b}\right)^{\alpha} .
\end{aligned}
$$

Their difference holds

$$
\begin{aligned}
Y_{\alpha}\left(S_{n}\right)-Y_{\alpha}\left(S_{a, b}\right)= & (n-1)\left((n-1)^{n-1}+1\right)^{\alpha} \\
& -(a-1)\left((a-1)^{a-1}+1\right)^{\alpha} \\
& -(b-1)\left((b-1)^{b-1}+1\right)^{\alpha} \\
& -\left(a^{a}+b^{b}\right)^{\alpha}>0 .
\end{aligned}
$$

The reason of the above last inequality can be easily determined as $n-1>a$ and $n-1>b$ which implies that $(n-1)^{n-1}>(a-1)^{a-1}$ and $(n-1)^{n-1}>(b-1)^{b-1}$. This further implies that $(n-1)^{n-1}+1>(a-1)^{a-1}+1$ and $(n-1)^{n-1}+1>(b-1)^{b-1}+1 \quad$ where $\alpha \geq 1$. Hence, $Y_{\alpha}\left(S_{n}\right)>Y_{\alpha}\left(S_{a, b}\right)$. This completes the proof.

In a graph $G$, if the weight of every vertex is fixed, let such weight be $r \geq 1$; then, we define

$$
Y_{\alpha, r}(G)=\sum_{u v \in E(G)}\left(d(u)^{d(u)}+d(v)^{d(v)}+2 r\right)^{\alpha} .
$$

Theorem 3. For any $\alpha \geq 1$ and $r \geq 1$, the unique tree of order $n$ having maximum value of $Y_{\alpha, r}(G)$ is $S_{n}$.

Proof. On contrary base, let $T$ be a tree having maximum value of $Y_{\alpha, r}(G)$, where $T$ is neither a star nor a double star say $S_{a, b}$. Then, diam $(T)$ is greater or equal to 4 . Then, there is a vertex in $T$, say $t$, that is adjacent to two vertices say $x$ and $y$, such that $d_{T}(x)=a, d_{T}(y)=b$, and $d_{T}(t)=c$ without loss of generality let $a$ and $b \geq 2$. Such a tree is shown in Figure 7 while considering that every vertex has the fixed weight. Furthermore, for $x$ and $y$, we can have that $N(x)-$ $t=\left\{x_{1}, x_{2}, \ldots, x_{a-1}\right\}$ and $N(y)-t=\left\{y_{1}, y_{2}, \ldots, y_{b-1}\right\}$. We can easily construct another tree $T^{*}$ from $T$ by eliminating the edges $y y_{1}, y y_{2}, \ldots, y y_{b-1}$ and adding new edges $x y_{1}, x y_{2}, \ldots, x y_{b-1}$; for this, we refer [11]. For our proof, we proceed as follows:

$$
\begin{aligned}
Y_{\alpha, r}\left(T^{*}\right)= & \sum_{i=1}^{a-1}\left((a+b-1)^{a+b-1}+d_{T^{*}}\left(x_{i}\right)^{d_{T^{*}}\left(x_{i}\right)}+2 r\right)^{\alpha} \\
& +\sum_{i=1}^{b-1}\left((a+b-1)^{a+b-1}+d_{T^{*}}\left(y_{i}\right)^{d_{T^{*}}\left(y_{i}\right)}+2 r\right)^{\alpha} \\
& +\left((a+b-1)^{a+b-1}+c^{c}+2 r\right)^{\alpha} \\
& +\left(c^{c}+1+2 r\right)^{\alpha} .
\end{aligned}
$$

On similar way for $T$, we have

$$
\begin{aligned}
Y_{\alpha, r}(T)= & \sum_{i=1}^{a-1}\left(a^{a}+d_{T}\left(x_{i}\right)^{d_{T}\left(x_{i}\right)}+2 r\right)^{\alpha} \\
& +\sum_{i=1}^{b-1}\left(b^{b}+d_{T}\left(y_{i}\right)^{d_{T}\left(y_{i}\right)}+2 r\right)^{\alpha} \\
& +\left(a^{a}+c^{c}+2 r\right)^{\alpha}+\left(c^{c}+b^{b}+2 r\right)^{\alpha} .
\end{aligned}
$$

As in Theorem 2, we can easily show that there is a contradiction to the maximality of $Y_{\alpha, r}(G)$, where $G=T$. Also, the following holds:

$$
\begin{aligned}
Y_{\alpha, r}\left(S_{n}\right)-Y_{\alpha, r}\left(S_{a, b}\right)= & (n-1)\left((n-1)^{n-1}+1+2 r\right)^{\alpha} \\
& -(a-1)\left((a-1)^{a-1}+1+2 r\right)^{\alpha} \\
& -(b-1)\left((b-1)^{b-1}+1+2 r\right)^{\alpha} \\
& -\left(a^{a}+b^{b}+2 r\right)^{\alpha}>0 .
\end{aligned}
$$

The last inequality can easily be determined as $n-1>a$ and $n-1>b$ which implies that $(n-1)^{n-1}>(a-1)^{a-1}$, $(n-1)^{n-1}>(b-1)^{b-1}$. This further implies that $(n-1)^{n-1}+1+2 r>(a-1)^{a-1}+1+2 r$ and $(n-1)^{n-1}+$ $1+2 r>(b-1)^{b-1}+1+2 r$, where $\quad \alpha \geq 1$. Hence, $Y_{\alpha, r}\left(S_{n}\right)>Y_{\alpha, r}\left(S_{a, b}\right)$.

Theorem 4. For $k \geq 1$, let $G \in T_{k}(n)$. If $Y_{\alpha}(G)$ is minimum and $w$ be a quasi-vertex of $G$, then $d(w)=2$.

Proof. Let $G \in T_{k}(n)$ such that $Y_{\alpha}(G)$ is minimum. We have to show that $d(w)=2$ where $w$ is quasi-vertex. On contrary base, suppose that $d(w)=1$. In this case, $w$ cannot be quasivertex because $w$ will be pendent vertex and $w$ will make no difference in the formation of quasi-tree. On the contrary, let $d(w) \geq 2$, and this shows that $w$ is adjacent to more than two 
vertices in $G$. Therefore, for elimination of any edge $w d \in E(G)$, we have $Y_{\alpha}(G)>Y_{\alpha}(G-w d)$ where $Y_{\alpha}(G-w d) \in T_{k}(n)$. This is contradiction to the minimality of $Y_{\alpha}(G)$. This contradiction is due to our wrong supposition that $d(w) \geq 2$. Hence, in both cases, there is contradiction due to which we are forced to accept that $d(w)=2$.

Theorem 5. Let $G \in T_{k}(n)$, where $k \geq 1$ and $n \geq 5 . \alpha \geq 1$ holds

$$
\begin{aligned}
Y_{\alpha}(G) \leq & 2^{\alpha-1} k(k+1)(n-1)^{\alpha \cdot(n-1)} \\
& +(k+1)(n-k-1)\left((n-1)^{(n-1)}+(k+1)^{(k+1)}\right)^{\alpha}
\end{aligned}
$$

where the equality is satisfied if and only if $G=K_{k}+S_{n-k}$.

Proof. Let $G \in T_{k}(n)$ and $\alpha \geq 1$ be such that $Y_{\alpha}(G)$ is maximum as possible. Let the set of quasi-vertex be represented by $V_{k} \subset V(G)$. By means of Lemmas 1 and 2, we have $G=K_{k}+T_{n-k}$, where $T_{n-k}$ is a tree of order $n-k$. We have to prove that $T_{n-k}=S_{n-k}$. For this, we have, from [4],

$$
\begin{aligned}
Y_{\alpha}(G)= & Y_{\alpha}\left(K_{k}+T_{n-k}\right)=\sum_{u v \in E\left(K_{k}\right)}\left(d_{G}(u)^{d_{G}(u)}+d_{G}(v)^{d_{G}(v)}\right)^{\alpha} \\
& +\sum_{u \in V\left(K_{k}\right) v \in V\left(T_{n-k}\right)}\left(d_{G}(u)^{d_{G}(u)}+d_{G}(v)^{d_{G}(v)}\right)^{\alpha} \\
& +\sum_{u v \in E\left(T_{n-k}\right)}\left(d_{G}(u)^{d_{G}(u)}+d_{G}(v)^{d_{G}(v)}\right)^{\alpha} .
\end{aligned}
$$

We calculate each sum separately:

$$
\begin{aligned}
& \sum_{u v \in E\left(K_{k}\right)}\left(d_{G}(u)^{d_{G}(u)}+d_{G}(v)^{d_{G}(v)}\right)^{\alpha} \\
= & 2^{\alpha-1} k(k-1) \cdot(n-1)^{\alpha \cdot(n-1)} .
\end{aligned}
$$

Similarly, the middle sum in (26) can be obtained as

$$
\begin{aligned}
& \sum_{\substack{u \in V\left(K_{k}\right) \\
v \in V\left(T_{n-k}\right)}}\left(d_{G}(u)^{d_{G}(u)}+d_{G}(v)^{d_{G}(v)}\right)^{\alpha} \\
= & k \sum_{v \in V\left(T_{n-k}\right)}\left((n-1)^{n-1}+\left(d_{T_{n-k}}(v)+k\right)^{d_{T_{n-k}}(v)+k}\right)^{\alpha} .
\end{aligned}
$$

Using Theorem 2, the sum in (28) attains its maximum if $T_{n-k}=S_{n-k}$. For this, the above last sum becomes

$$
\begin{aligned}
& \sum_{u \in V\left(K_{k}\right) v \in V\left(S_{n-k}\right)}\left(d_{G}(u)^{d_{G}(u)}+d_{G}(v)^{d_{G}(v)}\right)^{\alpha} \\
& =k(n-k-1)\left((n-1)^{(n-1)}+(k+1)^{(k+1)}\right)^{\alpha} \\
& +2^{\alpha} k(n-1)^{\alpha(n-1)} .
\end{aligned}
$$

Similarly, the last sum in (26) is calculated as follows:

$$
\begin{aligned}
& \sum_{u v \in E\left(T_{n-k}\right)}\left(d_{G}(u)^{d_{G}(u)}+d_{G}(v)^{d_{G}(v)}\right)^{\alpha} \\
= & \sum_{u v \in E\left(T_{n-k}\right)}\left(\left(d_{T_{n-k}}(u)+k\right)^{\left(d_{T_{n-k}(u)}+k\right)}\right. \\
& \left.+\left(d_{T_{n-k}}(v)+k\right)^{\left(d_{T_{n-k}}(v)+k\right)}\right)^{\alpha} .
\end{aligned}
$$

By means of Theorem 2, the above last sum will obtain its maximum if $T_{n-k}=S_{n-k}$. For this, we have the following:

$$
\begin{aligned}
& \sum_{u v \in E\left(S_{n-k}\right)}\left(\left(d_{S_{n-k}}(u)+k\right)^{\left(d_{S_{n-k}(u)}+k\right)}+\left(d_{S_{n-k}}(v)+k\right)^{\left(d_{S_{n-k}}(v)+k\right)}\right)^{\alpha} \\
= & (n-k-1)\left((1+k)^{(1+k)}+(n-1)^{(n-1)}\right)^{\alpha} .
\end{aligned}
$$

Combining (27), (29), and (31), we have $T_{n-k}=S_{n-k}$, and consequently, for $G=K_{k}+S_{n-k}$, we get the following:

$$
\begin{aligned}
Y_{\alpha}(G)= & 2^{\alpha-1} k(k+1)(n-1)^{\alpha \cdot(n-1)} \\
& +(k+1)(n-k-1)\left((n-1)^{(n-1)}+(k+1)^{(k+1)}\right)^{\alpha} .
\end{aligned}
$$

Theorem 6. Let $G \in T_{k}(n)$ and $\alpha \geq 1$.

(i) If $k=1$ and $n \geq 3$, then $Y_{\alpha}(G) \geq n \cdot 8^{\alpha}$. The equality $Y_{\alpha}(G)=n \cdot 8^{\alpha}$ holds if and only if $G=C_{n}$, where $C_{n}$ is a cycle of order $n$.

(ii) If $k=2$ and $n=4$, then $Y_{\alpha}(G) \geq 54^{\alpha}+4 \cdot 31^{\alpha}$. If $k=$ 2 and $n \geq 5$, then $Y_{\alpha}(G) \geq 6 \cdot 31^{\alpha}+(n-5) \cdot 8^{\alpha}$. Furthermore, the equality holds if and only if $G$ consists of two cycles of length three having a common edge for $n=4$ or two cycles with a common path having length at least two for $n \geq 5$ or two cycles joined by a path of length at least two for $n \geq 7$.

Proof. Suppose that $G \in T_{k}(n)$, where $Y_{\alpha}(G)$ is as small as possible. Let the set of quasi-vertices be $V_{k}$; then, by the definition of a $k$-generalized quasi-tree, $G-V_{k}$ is a tree having order $n-k$. By Theorem 5 , we have $d(s)=2$ for all $s \in V_{k}$. Furthermore, for every vertex $s$ in $V_{k}, s$ is adjacent to two vertices of $V-V_{k}$. From this discussion, we deduce that $G$ is a connected graph with $n+k-1$ edges. It means that $G$ has internal $k$-cycles. First of all, we have to show that $G$ has no pendent vertex. For this, suppose on contrary base that $G$ has a pendent vertex $x$. Let there be another vertex $z$ where there is a path between $x$ and $z$, and let also $z$ belong to a cycle, say $C$, in $G$. Let this path be $x, v, \ldots, z$. We denote the vertices adjacent to $v$ different from $x$ by $x_{1}, x_{2}, \ldots, x_{t}$, and let their degrees be $d\left(x_{i}\right)=d_{i}, 1 \leq i \leq t$. Then, we have $d_{G}(v)=t+1$. Obviously, $t \geq 1$ and at least one degree $d_{i} \geq 2$. We can define a new $k$-cyclic graph having order $n$, by deleting the edge between $x$ and $v$ and then arranging $x$ between two consecutive vertices, say $g$ and $h$, on the cycle $C$. 


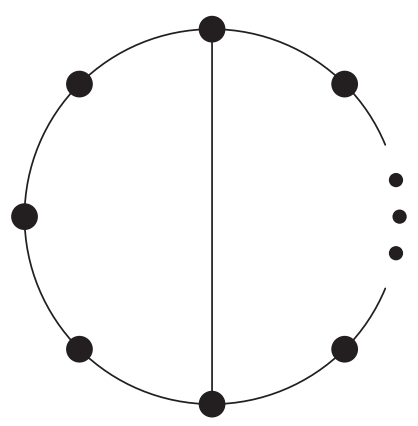

Figure 8: A graph for the degree sequence $d^{*}$ and $L=1$.

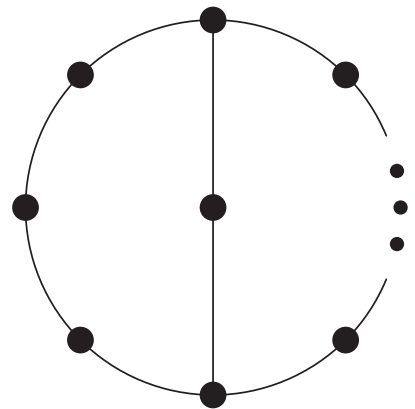

Figure 9: A graph for the degree sequence $d^{*}$ and $L=2$.

Let $d_{G}(g)=a$ and $d_{G}(h)=b$; clearly, $a$ and $b \geq 2$. Then, in the new graph, say $G_{*}$, the degree of $v$ is $t, d_{G_{*}}(g)=a$, $d_{G_{*}}(h)=b$, and $d_{G_{*}}\left(x_{i}\right)=d_{i}$ for every $1 \leq i \leq t$. We can write the difference of $Y_{\alpha}^{*}(G)-Y_{\alpha}\left(G_{*}\right)$ as

$$
\begin{aligned}
Y_{\alpha}(G)-Y_{\alpha}\left(G_{*}\right)= & \sum_{u v \in E(G)}\left(d_{G}(u)^{d_{G}(u)}+d_{G}(v)^{d_{G}(v)}\right)^{\alpha} \\
& -\sum_{e f \in E\left(G_{*}\right)}\left(d_{G_{*}}(e)^{d_{G_{*}}(e)}+d_{G_{*}}(f)^{d_{G_{*}}(f)}\right)^{\alpha} \\
= & \sum_{i=1}^{t}\left[\left((t+1)^{(t+1)}+d_{i}^{d_{i}}\right)^{\alpha}-\left(t^{t}+d_{i}^{d_{i}}\right)^{\alpha}\right] \\
& +\left((t+1)^{(t+1)}+1\right)^{\alpha} \\
& +\left(a^{a}+b^{b}\right)^{\alpha}-\left(a^{a}+2^{2}\right)^{\alpha}-\left(b^{b}+2^{2}\right)^{\alpha} .
\end{aligned}
$$

For $t \geq 2$, we have the following:

$$
\sum_{i=1}^{t}\left[\left((t+1)^{(t+1)}+d_{i}^{d_{i}}\right)^{\alpha}-\left(t^{t}+d_{i}^{d_{i}}\right)^{\alpha}\right]>0 .
$$

Also, $\left((t+1)^{(t+1)}+1\right)^{\alpha} \geq 28^{\alpha}$; by using Lemma 3 , we have $\quad\left(\left((t+1)^{(t+1)}+1\right)^{\alpha}+\left(a^{a}+b^{b}\right)\right)^{\alpha}-\left(a^{a}+2^{2}\right)^{\alpha}-$ $\left(b^{b}+2^{2}\right)^{\alpha}>0$, which shows that $Y_{\alpha}(G)-Y_{\alpha}\left(G_{*}\right)>0$. This is a contradiction to the minimality of $G$, that is, $G_{*}$ is another $k$-cyclic graph having $Y_{\alpha}\left(G_{*}\right)$ smaller than that of $Y_{\alpha}(G)$.

Now, for $t=1$, it implies that $v$ is a pendent vertex of $G_{*}$; by doing the same process for $v$ instead of $x$, we will

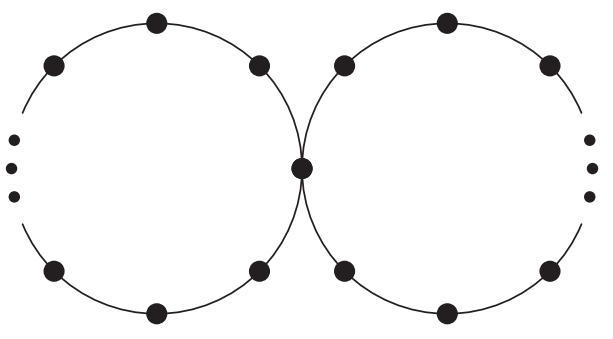

Figure 10: Graph for degree sequence $d^{* *}$.

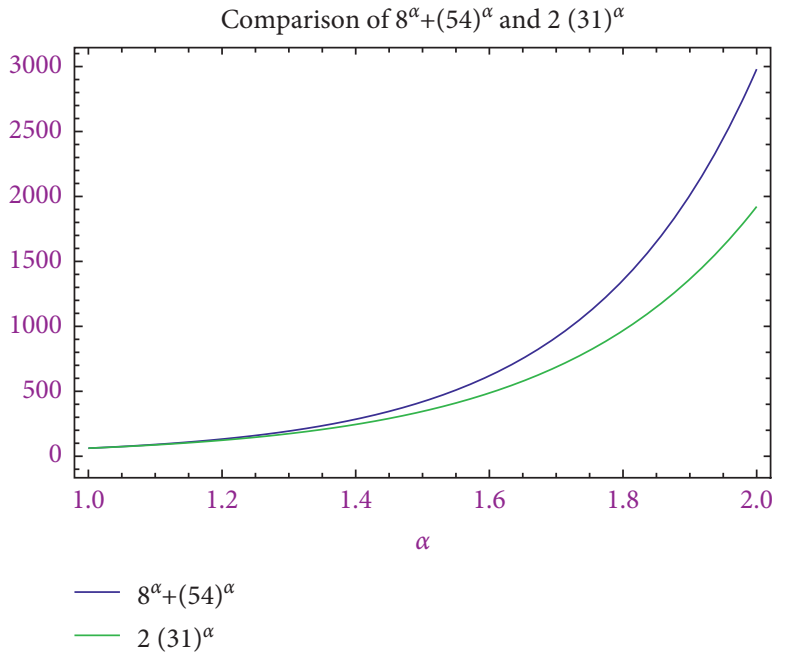

Figure 11: Plot for $A-B$, and it shows that $A>B$.

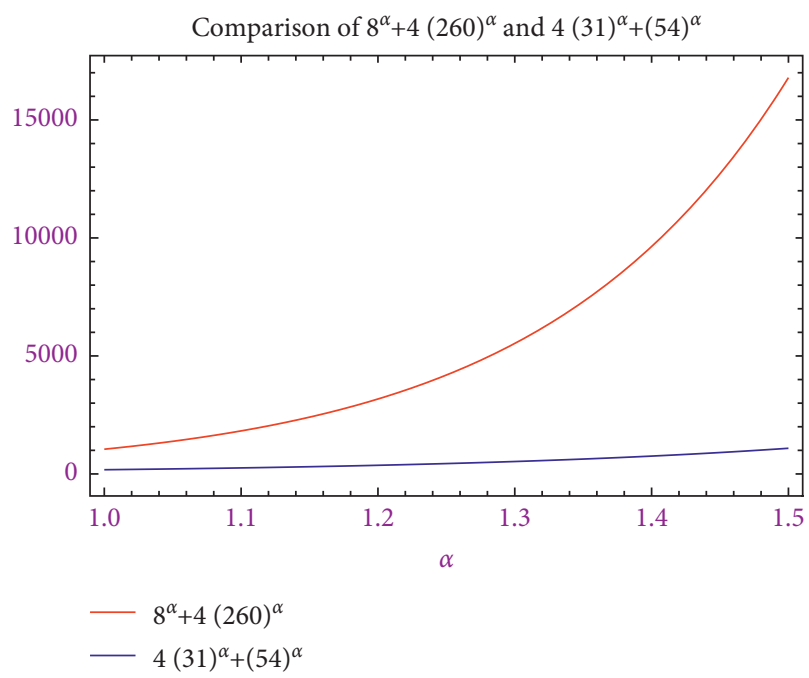

Figure 12: Plot for $C-A$, and it shows that $C>A$.

observe that there is a pendent vertex $v$ that is adjacent to $z$ on cycle $C$; then, $d(z) \geq 3$ in this case. We will again get a contradiction, and this contradiction is due to our wrong supposition; hence, we are forced to accept that $d(s) \neq 1$.

Next, we have to suppose that, for all $s \in V(G), d(s) \geq 2$. 
(i) Then, for $k=1$, this implies that $G$ is a connected graph having one cycle and $n$ edges. Obviously, $G=C_{n}$; thus, each vertex of $G$ has degree 2 , and then,

$$
Y_{\alpha}(G)=n \cdot 8^{\alpha}
$$

Otherwise, $Y_{\alpha}(G)$ will be greater than that of $n .8^{\alpha}$.

(ii) For $k=2$, it shows that $G$ is a 2-cyclic graph and has $n+1$ edges. Now, the sum of degrees of $G$ is given in the following:

$$
\sum_{v \in G} d(v)=2+2 n
$$

Let us denote these degree sequences by $d^{*}$ or $d^{* *}$, that is,

$$
d^{*}(G)=[3,3,2,2, \ldots, 2],
$$

or

$$
d^{* *}(G)=[4,2,2, \ldots, 2]
$$

If the degree sequence of $G$ is $d^{*}$, then

(a) The graph $G$ contains a common path of length $L \geq 1$, or

(b) The graph $G$ has two cycles joined by a path of length $L \geq 1$

In these cases, if $L=1$, then such graph is shown in Figure 8.

For this, we have

$$
Y_{\alpha}(G)=54^{\alpha}+4 \cdot 31^{\alpha}+(n-4) \cdot 8^{\alpha}=A .
$$

If $L=2$, then we have a graph illustrated in Figure 9 . For this graph, we obtain

$$
Y_{\alpha}(G)=6 \cdot 31^{\alpha}+(n-5) \cdot 8^{\alpha}=B
$$

If degree sequence of $G$ is $d^{* *}$, then in this case $G$ has two cycles having a vertex in common, as shown in Figure 10.

For this graph, we get

$$
Y_{\alpha}(G)=4 \cdot 260^{\alpha}+(n-3) \cdot 8^{\alpha}=C \text {. }
$$

From $A, B$, and $C$, we have $A>B$ which is equivalent to $8^{\alpha}+54^{\alpha}>2 \cdot 31^{\alpha}$ and is obvious which can be easily determined through Figure 11 . Now, $C>A$ which implies that $C-A$ is positive. This can be seen in Figure 12.

\section{Data Availability}

The data used to support the findings of the study are included within this paper.

\section{Conflicts of Interest}

The authors declare that there are no conflicts of interest.

\section{Authors' Contributions}

Rui Cheng wrote the final version of this paper and approved the results, Gohar Ali supervised this work, Gul Rahmat proved the results, Muhammad Yasin Khan wrote the paper, Andrea Semanicova-Fenovcikova plotted the results, and Jia-Bao Liu analyzed the results and arranged the funding for this paper.

\section{Acknowledgments}

This work was supported by the Slovak Research and Development Agency under the contract no. APVV-19-0153 and by VEGA 1/0233/18.

\section{References}

[1] M. K. Jamil, I. Tomescu, and M. Imran, "Extremal $k$-generalized quasi trees for general sum-connectivity index," UPB Scientific Bulletin, Series A, vol. 82, no. 2, pp. 101-106, 2020.

[2] M. Numan, S. I. Butt, and A. Taimur, "Super cyclic antimagic covering for some families of graphs," Open Journal of Mathematical Sciences, vol. 5, no. 1, pp. 27-33, 2021.

[3] H. F. M. Salih and S. M. Mershkhan, "Generalized the liouvilles and mbius functions of graph," Open Journal of Mathematical Sciences, vol. 4, no. 1, pp. 186-194, 2020.

[4] D. A. Mojdeh, M. Habibi, L. Badakhshian, and Y. Rao, "Zagreb indices of trees, unicyclic and bicyclic graphs with given (total) domination," IEEE Access, vol. 7, pp. 9414394149, 2019.

[5] L. Ding, Lei, S. A. Ul Haq Bokhary et al., "Degree-based indices of some complex networks," Journal of Mathematics, vol. 2021, Article ID 5531357, 16 pages, 2021.

[6] M. Liu, I. Tomescu, and I. Liu, "Unified extremal results for $k$-apex unicyclic graphs (trees)," Discrete Applied Mathematics, vol. 288, pp. 35-49, 2021.

[7] B. Basavanagoud and P. Jakkannavar, "Kulli-Basava indices of graphs," International Journal of Applied Engineering Research, vol. 14, no. 1, pp. 325-342, 2019.

[8] F. Asif, Z. Zahid, and S. Zafar, "Leap Zagreb and leap hyperZagreb indices of Jahangir and Jahangir derived graphs," Engineering and Applied Science Letter, vol. 3, no. 2, pp. 1-8, 2020.

[9] V. Pletser, "Congruence properties of indices of triangular numbers multiple of other triangular numbers," Open Journal of Mathematical Sciences, vol. 5, no. 1, pp. 262-269, 2021.

[10] H. M. Nagesh and V. R. Girish, "On the entire Zagreb indices of the line graph and line cut-vertex graph of the subdivision graph," Open Journal of Mathematical Sciences, vol. 4, no. 1, pp. $470-475,2020$.

[11] Y. Hu, X. Li, and Y. Yuan, "Trees with maximum general Randić index," MATCH Communications in Mathematical and in Computer Chemistry, vol. 52, pp. 129-146, 2004. 